\title{
CONNECTION SPACE APPROACH TO AMBIGUITIES OF GAUGE THEORIES
}

\author{
R. ALDROVANDI AND A. L. BARBOSA
}

Received 24 October 2004 and in revised form 17 July 2005

Two distinct gauge potentials can have the same field strength, in which case they are said to be "copies" of each other. The consequences of this ambiguity for the general affine space $\mathscr{A}$ of gauge potentials are examined. Any two potentials are connected by a straight line in $\mathscr{A}$, but a straight line going through two copies either contains no other copy or is entirely formed by copies.

\section{Introduction}

In an nonabelian gauge theory, two or more distinct gauge potentials can correspond to the same field strength. As only field strengths are measurable, it is impossible to know exactly which gauge potential is actually at work in a given physical system. This is the Wu-Yang ambiguity [26], whose importance comes from the fact that, in quantum field theory, the fundamental fields are the gauge potentials and not the field strengths. In particular, the corresponding particles are the quanta of the gauge potentials. A good understanding of this ambiguity, in all its aspects and consequences, does not seem to have been as yet achieved. Activity on the subject has been intensive in the first years after its discovery $[4,6,7,13,14,20,25]$ but declined afterwards [19]. Progress has been made step by step, sometimes through the discovery of general properties of formal character $[9,10]$, most of times by unearthing particular cases which elucidate special points [3].

This paper presents a formal property which shows up in the space of gauge potentials and provides (at least) a partial classification of the connections corresponding to a given curvature. We will use both the physicist's and the mathematician's nomenclatures interchangeably. It should perhaps be recalled that what physicists call "gauge potentials" and "field strengths" are, in the language of mathematicians, "connections" and "curvatures" on fiber bundles. They are, respectively, 1-forms and 2 -forms with values in the Lie algebra of the gauge group (the bundle "structure group"). Connections and curvatures belong, consequently, to the adjoint representation, in which the group acts on its own Lie algebra. We will use an invariant notation, such as $A=J_{a} A^{a}{ }_{\mu} d x^{\mu}$ for a connection related to a gauge group with generators $\left\{J_{a}\right\}, F=(1 / 2) J_{a} F^{a}{ }_{\mu \nu} d x^{\mu} \wedge d x^{\nu}$ for the field 
strength, $K=J_{a} K^{a}{ }_{\mu} d x^{\mu}$ for covectors in the adjoint representation of the gauge algebra, and so forth. Wedge products will be left implicit.

The Wu-Yang ambiguity is introduced in Section 2. Connections related to a fixed group constitute a space [23] which we will call "A-space," or $A$. The main properties of this space of gauge potentials are presented in Section 3. The ambiguity is recast into a more cogent language in Section 4. The discussion of the affine character of $\mathscr{A}$ given in Section 5 leads then to the main results, given in Section 6.

\section{General aspects}

Consider two connection forms $A$ and $A^{\sharp}$, with curvatures

$$
F=d A+A A, \quad F^{\sharp}=d A^{\sharp}+A^{\sharp} A^{\sharp} .
$$

Under a gauge transformation produced by a member of the gauge group represented by the matrix $g$ in the adjoint representation, the connections will transform noncovariantly according to

$$
A \Longrightarrow A^{\prime}=g A g^{-1}+g d g^{-1}, \quad A^{\sharp} \Longrightarrow A^{\sharp^{\prime}}=g A^{\sharp} g^{-1}+g d g^{-1} .
$$

The curvatures, on the other hand, are covariant under these transformations $F \Rightarrow F^{\prime}=$ $g F g^{-1}, F^{\sharp} \Rightarrow F^{\sharp^{\prime}}=g F^{\sharp} g^{-1}$. The difference

$$
K=A^{\sharp}-A
$$

will be a 1 -form transforming according to

$$
K^{\prime}=A^{\sharp^{\prime}}-A^{\prime}=g\left(A^{\sharp}-A\right) g^{-1}=g^{-1} K g .
$$

That is, the difference between two connections is a matrix covariant 1-form in the adjoint representation. The ambiguity appears when $F^{\sharp}=F$ but $K \neq 0$. Gauge potentials like $A$ and $A^{\sharp}$, corresponding to the same field strength, are usually called "copies."

\section{On the connection space $\mathscr{A}$}

The geometric background for any gauge theory is a principal bundle with the gauge group as structure group and (for relativistic field theories) space-time as base space. The principal bundle includes the basic actors (connections and curvatures), as its tangent structure contains the adjoint representation. Other ("source") fields, belonging to other representations, inhabit associated bundles. A "gauge" is a section on the principal bundle: it chooses, for each point $x$ of the base space, one proper point on the bundle. A section is taken into another by a gauge transformation, induced by a group element $g(x)$ which is distinct for distinct points of the base space. The group elements in a gauge theory are, thus, point-dependent.

It is then more convenient to take a functional point of view [11], and consider the mappings $g$ from the base space into the group. In the same token, each connection $A$ can be seen as the mapping taking point $x$ of the base space into its value $A(x)$. Space $\mathscr{A}$ 
is then the set of such mappings. The set $\mathscr{G}=\{g: x \rightarrow g(x)\}$ of all mappings from the base space into the group constitute the so-called "large group." Gauge covariance divides $\mathscr{A}$ into equivalence classes $[11,27]$, each class representing a potential up to gauge transformations as those given in (2.2). The space of gauge inequivalent connections is the quotient $\alpha=\mathscr{A} / \mathscr{G}$. An element of $\mathscr{A}$ can be locally represented by $A=(a, g)$, with $a \in \mathscr{A} / \mathscr{G}$ and $g \in \mathscr{G}$. More details on the $\mathscr{A}$-space structure can be found in [2]. For our purpose here, it will be sufficient to say that only variations along $\alpha=\mathscr{A} / \mathscr{G}$ are of interest for copies, as variations along $\mathscr{G}$ are mere gauge transformations.

\section{The ambiguity}

The ambiguity turns up in nonabelian theories because $F$, given as in (2.1), does not determine $A$. At each point of space-time, a gauge can be chosen in which $A=0$ and consequently $F=d A$. This is true also along a line $[15,16,17,18]$. One might think of integrating by the homotopy formula [21] to obtain $A$ from $F$. This is impossible, because the involved homotopy requires the validity of $F=d A$ on a domain of the same dimension of space-time and the alluded gauge cannot exist (unless $F=0$ ) on a domain of dimension 2 or higher [1]. For copies, the difference form $K$ defines a translation on space $\mathscr{A}$ leaving $F$ invariant. This invariance establishes another division of $\mathscr{A}$ into equivalence classes. In effect, define the relation $R$ by $A R A^{\sharp}$ if $A^{\sharp}$ is a copy of $A$. This relation is reflexive, transitive, and symmetric, consequently an equivalence. The space of connections with distinct curvatures will be the quotient $\alpha / R$.

Covariant differentials have different expressions for different representations and form degrees. For example, the gauge group element $g$ can be seen as a matrix acting on column vectors $V$ belonging to an associated vector representation. The covariant differentials according to $A$ and $A^{\sharp}$ will have, in that representation, the forms $D V=d V+A V$; $D^{\sharp} V=d V+A^{\sharp} V=d V+\left(A^{\ddagger}-A\right) V+A V$, from which follows that

$$
D^{\sharp} V=D V+K V \text {. }
$$

For a 1-form in the adjoint matrix representation, as the difference 1-form (2.3),

$$
D K=d K+A K+K A=d K+\{A, K\}, \quad D^{\sharp} K=d K+A^{\sharp} K+K A^{\sharp}=d K+\left\{A^{\sharp}, K\right\} .
$$

It is immediately found that

$$
D^{\sharp} K=D K+2 K K
$$

and the relation between the two curvatures is

$$
F^{\sharp}=F+D K+K K .
$$

A direct calculation gives

$$
D D K+[K, F]=0,
$$


which actually holds for any covariant 1-form in the adjoint representation. Equation (4.4) leads to a general result: given a connection $A$ defining a covariant derivative $D_{A}$, each solution $K$ of $D_{A} K+K K=0$ will give a copy.

\section{On the affine character of $\mathscr{A}$}

We have been making implicit use of one main property of the space $\mathscr{A}$ of connections, namely, $\mathscr{A}$ is a convex affine space, homotopically trivial [23]. One way to state this operationally [24] has been used above. Given a connection $A$, every other connection $A^{\#}$ can be written as $A^{\sharp}=A+K$, for some covariant covector $K$. Another way can be stated as follows: through any two connections $A$ and $A^{\sharp}$, there exists a straight line of connections $A_{t}$, given by

$$
A_{t}=t A^{\sharp}+(1-t) A \text {. }
$$

In this expression, $t$ is a real parameter, $A_{0}=A$, and $A_{1}=A^{\sharp}$. In terms of the difference form $K$, that straight line is written as

$$
A_{t}=A+t K=A^{\sharp}-(1-t) K .
$$

Of course, $d A_{t} / d t=K$. Indicating by $D_{t}$ the covariant derivative according to connection $A_{t}$, we find

$$
D_{t} K=D K+2 t K K
$$

The curvature of $A_{t}$ is

$$
F_{t}=d A_{t}+A_{t} A_{t}=t F^{\#}+(1-t) F+t(t-1) K K,
$$

or

$$
F_{t}=F+t D K+t^{2} K K=F+t D_{t} K-t^{2} K K
$$

Notice that $F_{0}=F, F_{1}=F^{\#}$. It follows that

$$
\frac{d F_{t}}{d t}=D K+2 t K K=D_{t} K
$$

\section{The copy structure of space $\mathscr{A}$}

The results of the previous section are valid for any two connections $A, A^{\sharp}$. We now address the question of copies. From (4.4), the necessary and sufficient condition to have $F^{\#}=F$ is

$$
D K+K K=0
$$

From the Bianchi identity $D^{\sharp} F^{\sharp}=0$ applied with $A^{\sharp}=A+K$, it follows that

$$
[K, F]=0 .
$$


These conditions [8] lead to the well-known determinantal conditions [5, 22], for the nonexistence of copies. Notice that (4.5) and (6.2) imply that $D D K=0$. Notice also en passant that copies are of interest only for nonabelian theories. In the abelian case, $K K \equiv$ $0, D K \equiv d K$, and condition (6.1) reduces to $d K=0$, which means that locally $K=d \phi$ for some $\phi$. Then $A^{\sharp}=A+d \phi$, a mere gauge transformation.

A first consequence of the conditions above is

$$
\frac{d F_{t}}{d t}=D_{t} K=(1-2 t) D K=(2 t-1) K K
$$

A second consequence is that now the line through $F$ and $F^{\sharp}$ takes the form

$$
F_{t}=F+t(t-1) K K=F+t(1-t) D K
$$

We have thus the curvatures of all the connections linking two copies along a line in connection space. Are there other copies on this line? In other words, is there any $s \neq 0,1$ for which $F_{s}=F$ ? The existence of one such copy would imply, by the two expressions in (6.4), that $D K=0$ and $K K=0$. But then, by the first equality of (5.5), all points on the line $A_{t}=A+t K$ are copies. Three colinear copies imply that $A_{t}$ is a line entirely formed of copies.

As $D K=0$ implies that $K K=0$ by (6.1), it also implies that $D^{\sharp} K=0$ (by (4.3)) and vice versa. Consequently, every point of the line $A_{t}=t A^{\sharp}+(1-t) A$ through two copies $A$ and $A^{\sharp}$ represents a copy when the difference tensor $K=A^{\sharp}-A$ is parallel-transported by either $A$ or $A^{\#}$.

In this case, $d F_{t} / d t=0$, that is, $F_{t}=F$ for all values of $t$. Also, $D_{t} K=0$ for all $t$, so that $K$ is parallel-transported by each connection on the line. Notice that an arbitrary finite $K$ such that $D K=0$ does not necessarily engender a line of copies. It is necessary that $K$ be a priori the difference between two copies.

The above condition is necessary and sufficient. If $F_{t} \neq F$ for some $t \neq 0,1,(6.4)$ implies both $D K \neq 0$ and $K K \neq 0$. If the line joining two copies includes one point which is not a copy, then all other points for $t \neq 0,1$ correspond to noncopies.

Given two copies and the straight line joining them, either there is no other copy on the line or every point of the line represents a copy.

As a consequence, if there are copies for a certain $F$, and one of them (say, $A$ ) is isolated, then there are no copies on the lines joining $A$ to the other copies. Notice, however, that the existence of families of copies dependent on continuous parameters is known [12]. Thus, certainly not every copy is isolated.

The question of isolated copies is better understood by considering, instead of the above finite $K$, infinitesimal translations on $\mathscr{A}$. In effect, consider the variation of $F$, $\delta F=d \delta A+\delta A A+A \delta A=D_{A} \delta A$. In order to have $\delta F=0$, it is enough that $D_{A} \delta A=0$. Consequently, no copy is completely isolated. There can be copies close to any $A$ : each variation satisfying $D_{A} \delta A=0$ leads to a copy. Taken together with what has been said above on the finite case, this means that there will be lines of copies along the "directions" of the parallel-transported $\delta A$ 's.

Notice that a line through copies of the vacuum is necessarily a line of copies. In effect, given $A$ and $A^{\sharp}$ with $F=F^{\sharp}=0$, there are a gauge in which $A=0$ and another gauge in 
which $A^{\sharp}=0$. Using the first of these gauges, $A_{t}=t K$ along the line. On the other hand, $F_{t}=t(t-1) K K=0$ by (6.4). As $D K+K K=0$, we can write $K K=D K+K K+K K=$ $d K+A K+K A+K K+K K=d K+A^{\sharp} K+K A^{\sharp}=D^{\sharp} K=D^{\sharp} A^{\sharp}=F^{\sharp}=0$. It follows that $F_{t}=0$.

Summing up, the overall picture is the following. From any $A$ will emerge lines of three kinds:

(i) lines of copies, given by those $\delta A$ which are parallel-transported by $A$;

(ii) lines of noncopies, given by those $\delta A$ which are not parallel-transported by $A$;

(iii) lines along covariant matrix 1 -forms $K$ satisfying $D_{A} K+K K=0$, which will meet one copy at $A+K$, and only that one.

\section{Acknowledgment}

The authors are thankful to Fundação de Amparo à Pesquisa do Estado de São Paulo (FAPESP), Brazil, and Conselho Nacional de Desenvolvimento Cientifico e Tecnológico (CNPq), Brazil, for financial support.

\section{References}

[1] R. Aldrovandi, P. B. Barros, and J. G. Pereira, The equivalence principle revisited, Found. Phys. 33 (2003), no. 4, 545-575.

[2] R. Aldrovandi and J. G. Pereira, An Introduction to Geometrical Physics, World Scientific, New Jersey, 1995.

[3] C. G. Bollini, J. J. Giambiagi, and J. Tiomno, Gauge field copies, Phys. Lett. B 83 (1979), no. 2, 185-187.

[4] L. S. Brown and W. L. Weisberger, Vacuum polarization in uniform non-Abelian gauge fields, Nuclear Phys. B 157 (1979), no. 2, 285-326.

[5] M. Calvo, Connection between Yang-Mills potentials and their field strengths, Phys. Rev. D (3) 15 (1977), no. 6, 1733-1735.

[6] S. Coleman, Non-Abelian plane waves, Phys. Lett. B 70 (1977), no. 1, 59-60.

[7] S. Deser and W. Drechsler, Generalized gauge field copies, Phys. Lett. B 86 (1979), no. 2, 189192.

[8] S. Deser and F. Wilczek, Non-uniqueness of gauge-field potentials, Phys. Lett. B 65 (1976), no. 4, 391-393.

[9] F. A. Doria, Quasi-abelian and fully nonabelian gauge field copies: a classification, J. Math. Phys. 22 (1981), no. 12, 2943-2951.

[10] _ The geometry of gauge field copies, Comm. Math. Phys. 79 (1981), no. 3, 435-456.

[11] L. D. Faddeev and S. Shatashvili, Algebraic and Hamiltonian methods in the theory of nonabelian anomalies, Theoret. and Math. Phys. 60 (1985), 770.

[12] D. Z. Freedman and R. R. Khuri, The Wu-Yang ambiguity revisited, Phys. Lett. B 329 (1994), no. 2-3, 263-270.

[13] M. B. Halpern, Field-strength formulation of quantum chromodynamics, Phys. Rev. D 16 (1977), no. 6, 1798-1801.

[14] Field-strength copies and action copies in quantum chromodynamics, Nuclear Phys. B 139 (1978), no. 4, 477-489.

[15] B. Z. Iliev, Normal frames and the validity of the equivalence principle. I. Cases in a neighbourhood and at a point, J. Phys. A 29 (1996), no. 21, 6895-6901.

[16] Normal frames and the validity of the equivalence principle. II. The case along paths, J. Phys. A 30 (1997), no. 12, 4327-4336. 
[17] Normal frames and the validity of the equivalence principle. III. The case along smooth maps with separable points of self-intersection, J. Phys. A 31 (1998), no. 4, 1287-1296.

[18] Normal frames for derivations and linear connections and the equivalence principle, J. Geom. Phys. 45 (2003), no. 1-2, 24-53.

[19] P. Majumdar and H. S. Sharatchandra, Gauge field copies, Phys. Rev. D (3) 63 (2001), no. 6, 067701, 4 pp.

[20] M. A. Mostow, The field copy problem: to what extent do curvature (gauge field) and its covariant derivatives determine connection (gauge potential)? Comm. Math. Phys. 78 (1980/81), no. 1, 137-150.

[21] C. Nash and S. Sen, Topology and Geometry for Physicists, Academic Press, London, 1987.

[22] R. Roskies, Uniqueness of Yang-Mills potentials, Phys. Rev. D (3) 15 (1977), no. 6, 1731-1732.

[23] I. M. Singer, Some remarks on the Gribov ambiguity, Comm. Math. Phys. 60 (1978), no. 1, 7-12.

[24] - The geometry of the orbit space for nonabelian gauge theories, Phys. Scripta 24 (1981), no. $5,817-820$.

[25] S. Solomon, On the field strength-potential connection in non-abelian gauge theory, Nuclear Phys. B 147 (1979), no. 1-2, 174-188.

[26] T. T. Wu and C. N. Yang, Concept of nonintegrable phase factors and global formulation of gauge fields, Phys. Rev. D (3) 12 (1975), no. 12, 3845-3857.

[27] Y. S. Wu and A. Zee, Abelian gauge structure inside nonabelian gauge theories, Nuclear Phys. B 258 (1985), no. 1, 157-178.

R. Aldrovandi: Instituto de Física Teórica, Universidade Estadual Paulista "Júlio de Mesquita Filho," Rua Pamplona 145, CEP 01405-900, São Paulo, Brazil

E-mail address: ra@ift.unesp.br

A. L. Barbosa: Instituto de Física Teórica, Universidade Estadual Paulista "Júlio de Mesquita Filho," Rua Pamplona 145, CEP 01405-900, São Paulo, Brazil

E-mail address: analucia@ift.unesp.br 


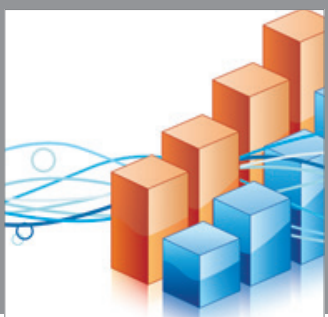

Advances in

Operations Research

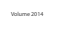

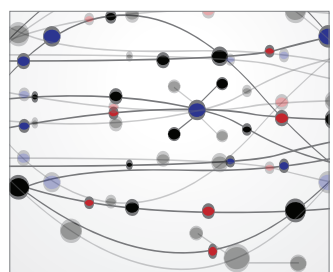

\section{The Scientific} World Journal
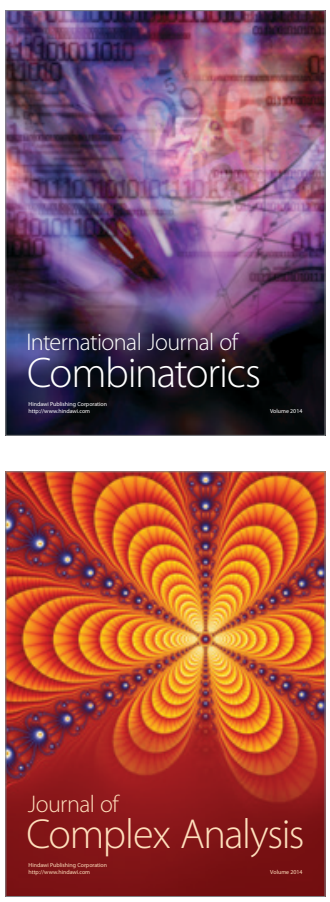

International Journal of

Mathematics and

Mathematical

Sciences
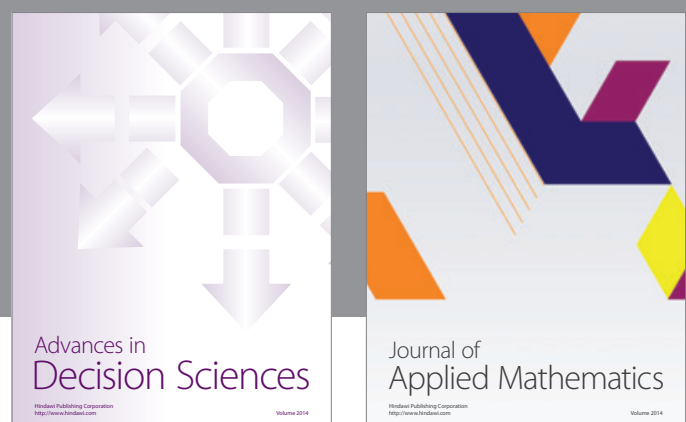

Journal of

Applied Mathematics
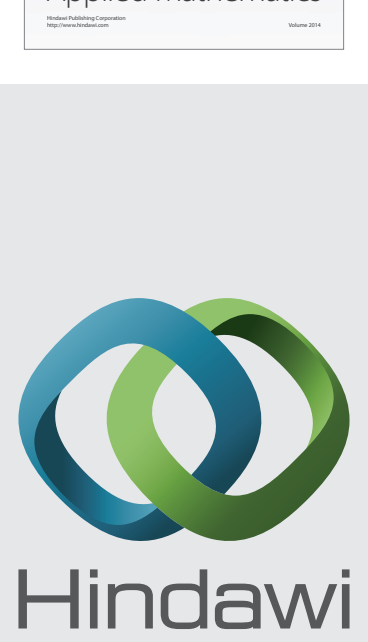

Submit your manuscripts at http://www.hindawi.com
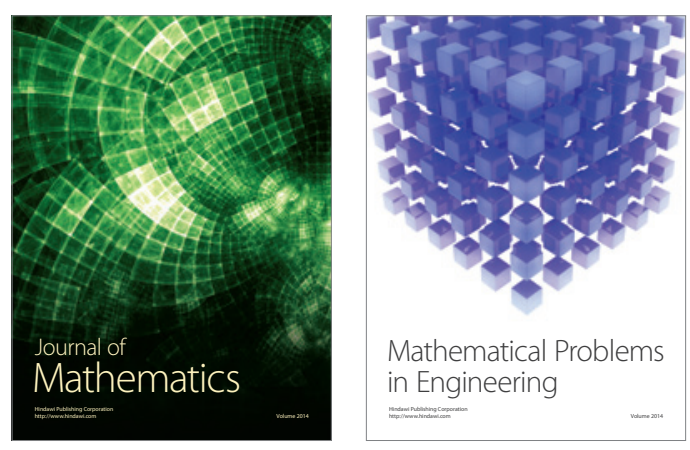

Mathematical Problems in Engineering
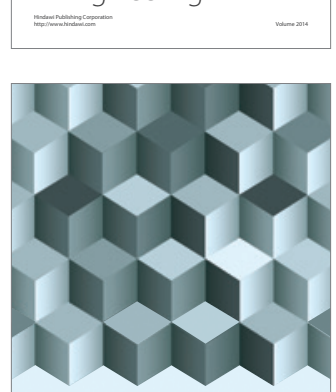

Journal of

Function Spaces
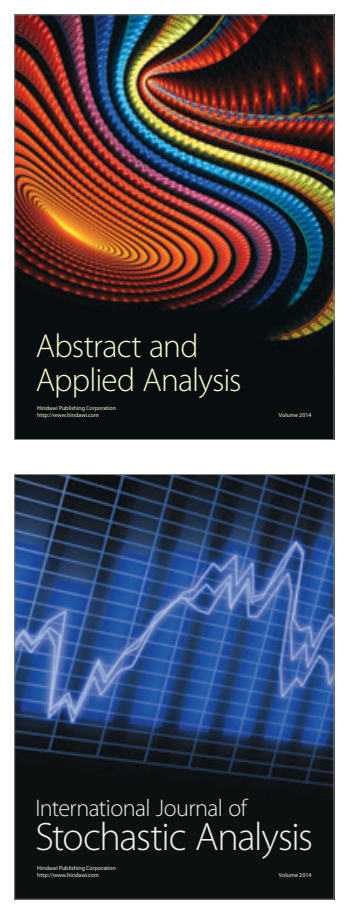

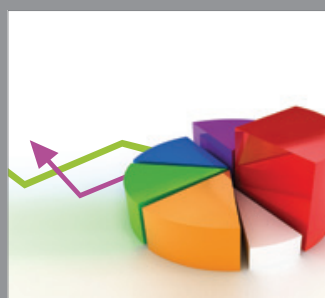

ournal of

Probability and Statistics

Promensencen
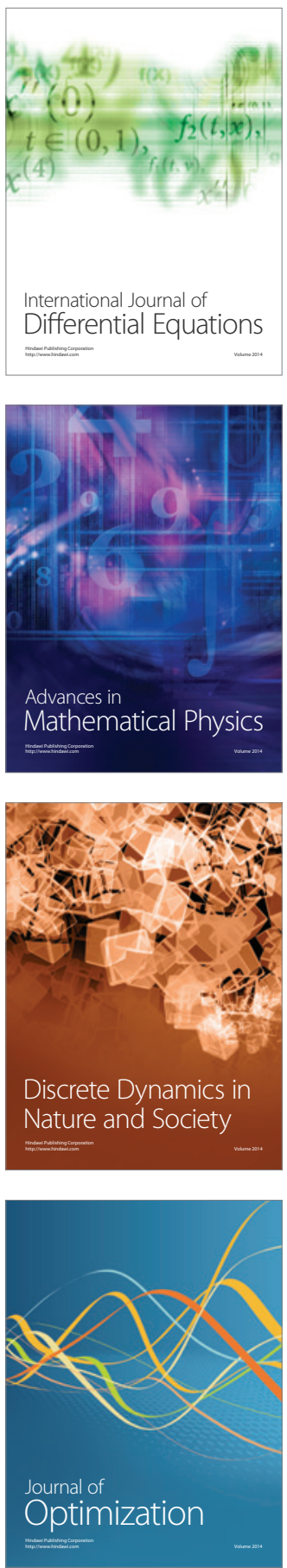